\title{
CENPF promotes papillary thyroid cancer progression by mediating cell proliferation and apoptosis
}

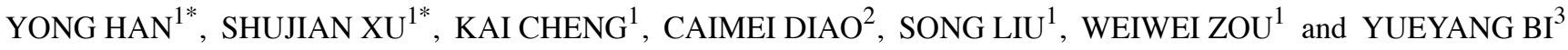 \\ ${ }^{1}$ Department of Breast and Thyroid Surgery, Binzhou Medical University Hospital, Binzhou, Shandong 256603; \\ ${ }^{2}$ Department of Health Management, The People's Hospital of South District of Qingdao, Qingdao, Shandong 266000; \\ ${ }^{3}$ Department of Respiratory Medicine, Binzhou Medical University Hospital, Binzhou, Shandong 256603, P.R. China
}

Received February 21, 2020; Accepted August 26, 2020

DOI: $10.3892 /$ etm.2021.9832

\begin{abstract}
Papillary thyroid cancer (PTHCA) accounts for $\sim 85 \%$ cases of thyroid cancer and exhibits high incidence. Targeted therapy is an effective method to combat this disease; however, novel therapeutic targets are required. Centromere protein $\mathrm{F}$ (CENPF), a member of centromere proteins and a transient kinetochore protein, regulates various cellular processes such as cell migration and mitosis, and its upregulation has been observed in multiple types of cancer, including breast cancer and gastric cancer. However, the potential role of CENPF in PTHCA progression is remains unclear. The results of the current study demonstrated that CENPF expression was enhanced in human PTHCA tissues through IHC assays. Furthermore, the expression of CENPF was correlated with the prognosis and the clinicopathological features, including $\mathrm{T}$ stage $(\mathrm{P}=0.021)$ and intraglandular dissemination $(\mathrm{P}=0.042)$ in patients with PTHCA. CENPF regulated the proliferation, apoptosis and cell cycle of PTHCA cells in vitro, which was confirmed through colony formation, MTT and flow cytometry assays, and affected tumor growth in vivo in mice. In conclusion, the current study reported the involvement of CENPF in PTHCA progression and provided a promising therapeutic target for PTHCA treatment.
\end{abstract}

\section{Introduction}

Thyroid cancer (THCA) is a disease with high incidence, low malignancy and slow tumor growth (1). Papillary thyroid cancer (PTHCA) accounts for $~ 85 \%$ of THCAs and is highly differentiated and with good therapeutic effects (2). While

Correspondence to: Dr Yueyang Bi, Department of Respiratory Medicine, Binzhou Medical University Hospital, 661 Huanghe Second Road, Binzhou, Shandong 256603, P.R. China

E-mail: hy797980@sina.cn

${ }^{*}$ Contributed equally

Key words: centromere protein F, papillary thyroid cancer, proliferation, apoptosis, cell cycle, therapeutic target early PTCHA does not have any obvious symptoms, the disease exhibits high invasion and metastasis at advanced stages such that surgical resection and chemoradiotherapy are not fully effective (3). Targeted therapy is an effective method to combat PTCHA and novel therapeutic targets are required.

Centromere protein $\mathrm{F}$ (CENPF) is a transient kinetochore protein that regulates multiple cellular processes, including chromosome segregation during mitosis $(4,5)$. The absence of CENPF causes various physical and developmental defects, including loss of CENF in mouse embryos, resulting in developmental failure (6-9). CENPF is upregulated during the $\mathrm{G} 2 / \mathrm{M}$ phase, inducing microtubule attachment and regulating cellular functions $(6,10)$. Additionally, CENPF interacts with several key cell cycle checkpoint proteins and late telophase proteins, including syntaxin 4 and synaptosomal-associated protein 25 , to further affect cellular processes $(11,12)$.

CENPF upregulation has been observed in multiple types of cancer, including hepatocellular carcinoma (HCC), breast cancer, nasopharyngeal cancer, prostate cancer and gastric cancer (11-15). The involvement of CENPF in cancer progression and metastasis, and its effects on prognosis, have been widely reported (16-19). CENPF was associated with the prognosis of non-small cell lung cancer (NSCLC) and prostate cancer (15-18). Additionally, CENPF overexpression was associated with poor prognosis and bone metastasis of breast cancer (19). Furthermore, a bioinformation analysis revealed an association between CENPF expression and the progression of non-muscle invasive bladder cancer (10). However, the potential role of CENPF in PTHCA progression remains unclear.

The present study aimed to clarify the role of CENPF in PTHCA progression. The current study demonstrated that CENPF upregulation in human PTHCA tissues and CENPF expression was correlated with the prognosis and clinical features in patients PTHCA. The effects of CENPF on PTHCA cell proliferation and apoptosis were further investigated. CENPF may be a promising molecular target for PTHCA treatment.

\section{Materials and methods}

Bioinformatic analysis. Bioinformatic analysis was performed using Gene Expression Profiling 
Interactive Analysis (http://gepia.cancer-pku.cn/detail. php?gene $=\mathrm{CENPF} /$ ) to analyze The Cancer Genome Atlas (TCGA; https://www.cancer.gov/about-nci/organization/ccg/research/structural-genomics/tcga) data with a threshold of $\mathrm{P}<0.05$ and $\operatorname{LogFC}>1$ or $<-1$ for differential genes. The median of survival rates was used as the basis for dividing patients into two groups for Kaplan-Meier survival analysis.

Antibodies, primers and short hairpin (sh)RNA plasmids. Anti-CENPF [for immunohistochemical (IHC) assays, 1:500; for immunoblot assays, 1:2,000; cat. no. ab84697], anti- $\beta$-actin $(1: 2,000$; cat. no. ab8226), horseradish peroxidase (HRP)-conjugated goat anti-rabbit IgG (1:5,000; cat. no. ab6721) and HRP-conjugated rabbit anti-mouse IgG (1:5,000; cat. no. ab6728) were purchased from Abcam.

Quantitative PCR (qPCR) primer sequences were as follows: CENF forward, 5'-TCTGCTCGGGTTCAAACT GG-3' and reverse, 5'-TGTGAGTCCGTGACCGAGTA-3'; and GAPDH forward, 5'-CGACCACTTTGTCAAGCTCA-3' and reverse, 5'-GGTTGAGCACAGGGTACTTTATT-3'. CENPF shRNA plasmids (cat. no. sc-37563-V) were purchased from Santa Cruz Biotechnology Co., Ltd.

Human tissue samples and analysis. A total of 87 human PTHCA tissues and corresponding normal tissues were collected from patients with PTHCA at Binzhou Medical University Hospital (Binzhou, China). Patients were divided into CENPF low expression and high expression groups according to CENPF expression in tumor tissues. Clinicopathological characteristics, including patient age, sex, tumor (T) stage (15), lymph node metastasis and intraglandular dissemination, are presented in Table I. All procedures performed in the current study were approved by the Ethics Committee of Binzhou Medical University Hospital (Binzhou, China). Written informed consent was obtained from all patients or their families.

IHC assays. To explore the possible association between CENPF expression and PTHCA progression, IHC assays were performed. Tissue sections (5- $\mu \mathrm{m}$ thickness) were fixed with $4 \%$ paraformaldehyde at room temperature for $20 \mathrm{~min}$ and blocked with 2\% BSA (Sigma-Aldrich; Merck KGaA) for $20 \mathrm{~min}$ at room temperature. Slides were incubated with CENPF antibodies for $2 \mathrm{~h}$ at room temperature. Sections were then incubated with mice or rabbit biotinylated secondary antibodies (1:5,000; cat. nos. ab6721 and ab6728; Abcam) for $1.5 \mathrm{~h}$ at room temperature. Diaminobenzidine (Sigma-Aldrich; Merck $\mathrm{KGaA}$ ) was used as a chromogen substrate. A light microscope (IX71; Zeiss AG) was used for imaging at x100 and x200 magnification.

IHC assays showed that CENPF was located in the cytoplasm of cells in PTHCA tissues. The scoring method used to analyze the proportion of positive stained cells was as follows: 0 (0\% stained cells), 1 (1-20\% stained cells), 2 (21-60\% stained cells) and 3 (61-100\% stained cells).

Staining intensity was evaluated on a score of 0 (no staining or low-level staining), 1 (modest staining) and 2 (high staining). CENPF expression levels were divided according to the staining index: Score of staining intensity + score of staining cells percentage. A staining index $<2$ was considered as low CENPF expression and $\geq 2$ was considered as high CENPF expression.

Cell culturesandtransfections. Human PTHCAcell lines TPC-1 and KTC-1 were purchased from the American Type Culture Collection. TPC-1 and KTC-1 cells were incubated in DMEM supplemented with 10\% FBS (Gibco; Thermo Fisher Scientific, Inc.) at $37^{\circ} \mathrm{C}$ in a $5 \% \mathrm{CO}_{2}$ incubator for $48 \mathrm{~h}$. CENPF shRNA plasmids $(1 \mu \mathrm{g} / \mu \mathrm{l}$; 5'-GTTTCAGCTTGACAGTCTCG-3') were transfected into TPC-1 and KTC-1 cells using Lipofectamine ${ }^{\circledR}$ 2000 (cat. no. 11668019; Invitrogen, Thermo Fisher Scientific, Inc.) for $6 \mathrm{~h}$. In 6-well plates, $5 \mu \mathrm{l}$ transfection reagent and $1 \mu \mathrm{g}$ plasmids were mixed in $250 \mu$ l of serum-free DMEM, left to stand for $5 \mathrm{~min}$ and then mixed. Following incubation at room temperature for $20 \mathrm{~min}$, the mix was added to serum-starved cells and incubated at $37^{\circ} \mathrm{C}$ for $4 \mathrm{~h}$. For the control group, the shRNA targeting sequence was nonsense and did not target intracellular RNAs. Only TPC-1 cells were used in the animal assays, and stable CENPF-depleted cells were used for the in vivo assays. Stable CENPF-depleted TPC-1 cells were screened through CENPF shRNA lentivirus infection to stably deplete CENPF expression and used for in vivo tumor growth assays.

qPCR assays. Trizol ${ }^{\circledR}$ (cat. no. 15596026; Invitrogen, Thermo Fisher Scientific, Inc.) was used to extract total RNA from human PTHCA cells. RNA was reverse-transcribed using M-MLV reverse transcriptase (cat. no. M1701; Promega Corporation). Total mRNA was reverse transcribed into cDNA by using a cDNA synthesis system (cat. no. 6110A; Takara Bio, Inc.) at $42^{\circ} \mathrm{C}$ for $1 \mathrm{~h}$. qPCR was performed using a SYBR Ex Taq kit (cat. no. 638319; Takara Bio, Inc.). The following thermocycling conditions were used for the qPCR: Initial denaturation at $95^{\circ} \mathrm{C}$ for $3 \mathrm{~min}$; followed by 30 cycles of denaturation at $95^{\circ} \mathrm{C}$ for $30 \mathrm{sec}$, annealing at $58^{\circ} \mathrm{C}$ for $30 \mathrm{sec}$ and extension a $72^{\circ} \mathrm{C}$ for $30 \mathrm{sec}$. The $2^{-\Delta \Delta \mathrm{Cq}}$ method was used to quantify results (20). CENPF expression levels were normalized to GAPDH.

Western blotting. PTHCA cells or tissues were lysed using RIPA buffer (cat. no. 9800; Cell Signaling Technology, Inc.). The BCA method was used for protein determination. Proteins $(20 \mathrm{mg} / \mathrm{lane})$ were separated via $8 \%$ SDS-PAGE. Proteins were then transferred onto PVDF membranes. Membranes were blocked with 5\% milk in TBS-T at $37^{\circ} \mathrm{C}$ for $2 \mathrm{~h}$ and incubated with the aforementioned primary antibodies for the detection of CENPF and $\beta$-actin at room temperature for $2 \mathrm{~h}$. Membranes were subsequently incubated with HRP-conjugate secondary antibodies for $1 \mathrm{~h}$ at room temperature. Signals were detected using an ECL kit (Novex ${ }^{\mathrm{TM}}$ Chemiluminescent Substrate Reagent kit; Thermo Fisher Scientific, Inc.) according to the manufacturer's protocol. Signal intensity was measured using ImageJ (version 1.8.0; National Institutes of Health).

Colony formation assays. PTHCA cells were plated into 6-well culture plates and transfected with control or CENPF shRNA plasmids as aforementioned. DMEM was replaced with fresh medium every 3 days. After 14 days, cells were fixed with $4 \%$ paraformaldehyde for $30 \mathrm{~min}$ at room temperature, stained 
Table I. Correlation between CENPF expression and clinicopathological features of 87 patients with papillary thyroid cancer.

\begin{tabular}{|c|c|c|c|c|c|}
\hline \multirow[b]{2}{*}{ Clinicopathological feature } & \multirow[b]{2}{*}{ No. of patients $(n=87)$} & \multicolumn{2}{|c|}{ CENPF expression } & \multirow[b]{2}{*}{$\chi^{2}$} & \multirow[b]{2}{*}{ P-value } \\
\hline & & Low $(n=22)$ & High $(\mathrm{n}=65)$ & & \\
\hline Age (years) & & & & 2.213 & 0.137 \\
\hline$<45$ & 32 & 11 & 21 & & \\
\hline$\geq 45$ & 55 & 11 & 44 & & \\
\hline Sex & & & & 3.804 & 0.051 \\
\hline Male & 22 & 9 & 13 & & \\
\hline Female & 65 & 13 & 52 & & \\
\hline T stage & & & & 5.368 & $0.021^{\mathrm{a}}$ \\
\hline $\mathrm{T}_{1}-\mathrm{T}_{2}$ & 37 & 14 & 23 & & \\
\hline $\mathrm{T}_{3}-\mathrm{T}_{4}$ & 50 & 8 & 42 & & \\
\hline Lymph node metastasis & & & & 0.035 & 0.851 \\
\hline Yes & 42 & 11 & 31 & & \\
\hline No & 45 & 11 & 34 & & \\
\hline Intraglandular dissemination & & & & 4.148 & $0.042^{\mathrm{a}}$ \\
\hline Yes & 40 & 6 & 34 & & \\
\hline No & 47 & 16 & 31 & & \\
\hline
\end{tabular}

${ }^{\mathrm{a}} \mathrm{P}<0.05$. CENPF, centromere protein $\mathrm{F}$; $\mathrm{T}$, tumor.

with $0.1 \%$ crystal violet at room temperature for $30 \mathrm{~min}$ and washed with PBS. Numbers of colonies were counted manually. A light microscope (IX71; Zeiss AG) was used for imaging at $\mathrm{x} 50$ magnification.

MTT assays. PTHCA cells were plated into 96-well plates (seeding density, $1 \times 10^{3} /$ well), transfected with control or CENPF shRNA plasmids and incubated for $24 \mathrm{~h}$. Cells were then incubated with MTT for $4 \mathrm{~h}$ at room temperature and DMEM was removed. Following this, $150 \mu 1$ DMSO was added into each well to extract stained cells. Optical density (OD) values were measured using a microplate reader at $570 \mathrm{~nm}$.

Cell cycle and apoptosis assays. An Annexin V-FITC Apoptosis Detection kit was purchased from Beijing Solarbio Science \& Technology Co., Ltd. (cat. no. CA1020). For cell apoptosis assays, PTHCA cells were fixed with $70 \%$ ethyl alcohol for $24 \mathrm{~h}$ at $-20^{\circ} \mathrm{C}$ and treated with Annexin V-FITC and propidium iodide (PI) for $20 \mathrm{~min}$ at room temperature. Cells were then analyzed using a FACS Calibur flow cytometer (FACSAria III; BD Biosciences).

For cell cycle assays, PTHCA cells were fixed with $70 \%$ ethyl alcohol for $24 \mathrm{~h}$ at $-20^{\circ} \mathrm{C}$ and incubated with $50 \mu \mathrm{g} / \mathrm{ml}$ $\mathrm{PI}$ at $37^{\circ} \mathrm{C}$ for $30 \mathrm{~min}$. The percentage of cells in different phases was analyzed using a flow cytometer (FACSAria III; BD Biosciences).

Tumor growth assays. All animal experiments were approved by the Institutional Animal Use Committee of Binzhou Medical University Hospital, Tianjin, China. Female BALB/c nude mice (8-week-old; $20 \mathrm{~g}$ ) were supplied by Beijing Vital River Experimental Animal Technology Co., Ltd.). Mice were fed with food and water ad libitum and were fed at specific pathogen-free conditions at $20^{\circ} \mathrm{C}, 60 \%$ humidity and alternating 12-h light/dark cycles. A total of 12 athymic nude mice were included in control $(n=6)$ and shRNA $(n=6)$ groups. TPC-1 cells were stably infected with control or CENPF shRNA lentivirus as aforementioned. Subsequently, $\sim 1 \times 10^{6}$ TPC-1 cells infected with the indicated lentivirus were subcutaneously injected into the abdomen of athymic nude mice. No mice died during the experiment. Mice were sacrificed via cervical dislocation prior to the removal of tumor tissue. Heartbeats were assessed to determine death and adequate humanitarian care was given.

At 15 days post-injection, tumors began to form, and tumor volumes were measured every 3 days. After 30 days, tumors were excised and photographed. Tumor growth curves were calculated and compared. Tumors were isolated and photographed, and tumor growth curves were calculated and compared. The tumor volume was calculated using the following formula: Tumor volume $\left(\mathrm{mm}^{3}\right)=$ tumor length $(\mathrm{mm})$ $\mathrm{x}$ tumor width $(\mathrm{mm})^{2} / 2$. In terms of weight, mice gained no more than $4 \%$ and lost no more than $10 \%$ of their body weight. The weights of different groups of mice were not significantly different between groups during the whole feeding period. The maximum diameter of tumors was $10 \mathrm{~mm}$ and the maximum tumor weight was $5 \%$ of the total body weight.

Statistical analysis. GraphPad software (version 6.0; GraphPad Software, Inc.) was used for statistical analysis. Data are presented as the mean \pm standard deviation. Associations between clinical features of patients with PTHCA and CENPF expression levels were analyzed by Pearson's $\chi^{2}$ test. Student's t-test was used for statistical comparison between two groups. $\mathrm{P}<0.05$ was considered to indicate a statistically significant difference. 
A

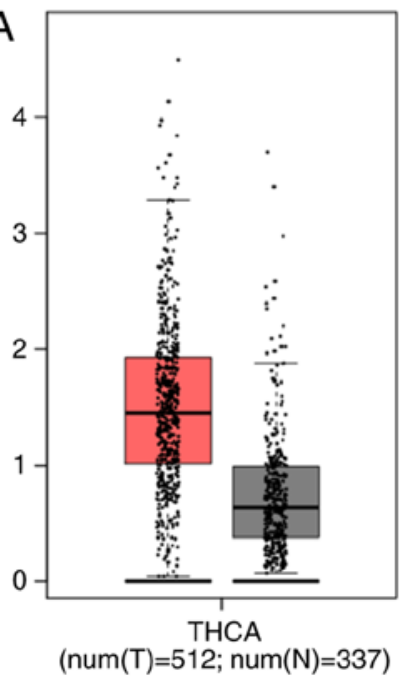

B

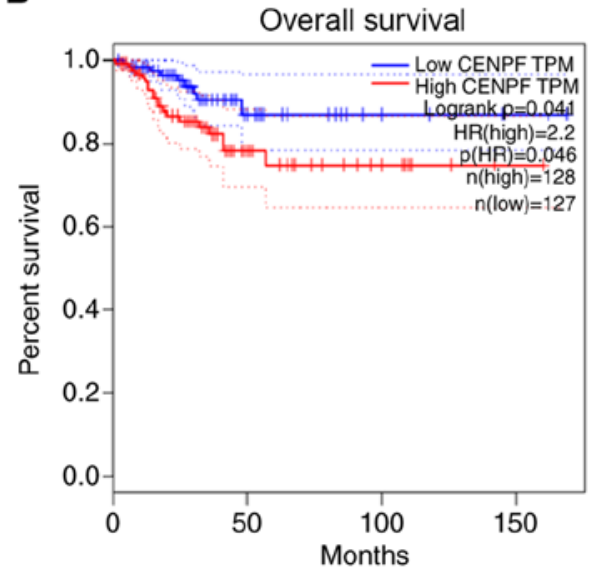

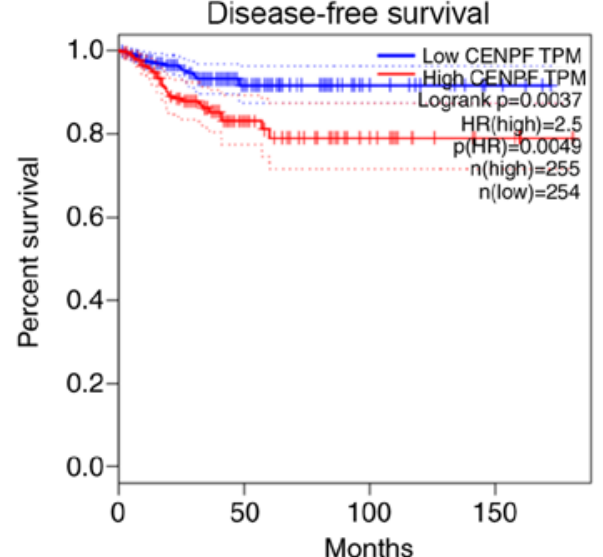

Figure 1. CENPF mRNA levels are increased in tumor tissues and are associated with the prognosis of patients with PTHCA. (A) CENPF mRNA levels in PTHCA and normal tissues according to the TCGA database. (B) CENPF expression was associated with the overall survival and disease-free survival rates of patients with PTHCA according to the TCGA database. The dotted line indicates the 95\% confidence interval. CENPF, centromere protein F; PTHCA, papillary thyroid cancer; TCGA, The Cancer Genome Atlas; TPM, transcripts perkilobase million; HR, hazard ratio.

\section{Results}

CENPF expression is enhanced in human PTHCA tissues and is associated with the prognosis and clinical features of patients. Relative mRNA levels of CENPF in human PTHCA tissues were compared with normal tissues using the TCGA database. According to the comparison between 512 tumor tissues and 337 normal tissues, high mRNA levels of CENPF were observed in PTHCA tissues (Fig. 1A). Furthermore, survival analysis of the database revealed that mRNA levels of CENPF were associated with overall survival and disease-free survival rates in patients with PTHCA (Fig. 1B), indicating an association between CENPF expression and patient prognosis.

Human PTHCA tissues exhibit high CENF expression in human PTHCA tissues. IHC assays were performed to investigate CENPF protein levels in PTHCA tissues and corresponding normal tissues. The results demonstrated that the expression of CENPF was markedly higher in PTHCA tissues (Fig. 2A). Furthermore, low CENPF expression was observed in corresponding normal tissues (Fig. 2B). These results confirmed high CENPF expression in human PTHCA tissues.

CENPF expression is associated with the clinicopathological features of patients with PTHCA. The clinicopathological features of 87 patients with PTHCA were analyzed. Patients were divided into CENPF low expression and high expression groups according to CENPF expression in tumor tissues. The results demonstrated that $22(25.3 \%)$ patients exhibited CENPF low expression and the remaining $65(74.7 \%)$ exhibited high expression (Table I). Clinicopathological features, including age, sex, T stage, lymph node metastasis and intraglandular dissemination, were analyzed. There was no association between CENPF expression and age $(\mathrm{P}=0.137)$, sex $(\mathrm{P}=0.051)$ and lymph node metastasis $(\mathrm{P}=0.851)$ in patients with PTHCA.
However, CENPF expression was associated with $\mathrm{T}$ stage $(\mathrm{P}=0.021)$ and intraglandular dissemination $(\mathrm{P}=0.042)$ in patients with PTHCA. The results revealed that CENPF expression was associated with certain clinicopathological features in patients with PTHCA.

CENPF depletion suppresses proliferation, stimulates apoptosis and causes cell cycle arrest in PTHCA cells. To further assess the involvement of CENPF in PTHCA progression, shRNA plasmids targeting CENPF were used to deplete CENPF expression in human PTHCA cell lines TPC-1 and KTC-1. Silencing efficiency was detected using qPCR assays (Fig. 3A) and western blotting (Fig. 3B). CENPF mRNA and protein levels were significantly decreased following CENPF depletion.

CENPF depletion results in proliferation defects, cell cycle arrest and apoptosis. MTT assays and colony formation assays were performed to assess the effects of CENPF on cytotoxicity in TPC-1 and KTC-1 cells. CENPF depletion resulted in significantly decreased colony numbers in TPC-1 and KTC-1 cells, as evidenced by colony formation assays (Fig. 4A). Furthermore, MTT assays demonstrated that relative OD values were significantly decreased in TPC-1 and KTC-1 cells following CENPF depletion, indicating defects in PTHCA cell cytotoxicity (Fig. 4B). The effects of CENPF on apoptosis and the cell cycle of TPC-1 and KTC-1 cells transfected with control or CENPF shRNA plasmids were detected using flow cytometry (FCM). The results demonstrated that CENPF depletion increased the percentage of apoptotic cells in TPC-1 and KTC-1 cells (Fig. 4C). Additionally, CENPF depletion led to the arrest of cell cycles in PTHCA cells, with the increase proportion of cells at the G1 phase (Fig. 4D). In summary, the results demonstrated the CENPF depletion resulted in proliferation defects and cell cycle arrest and stimulated the apoptosis of thyroid cancer cells in vitro. 
A

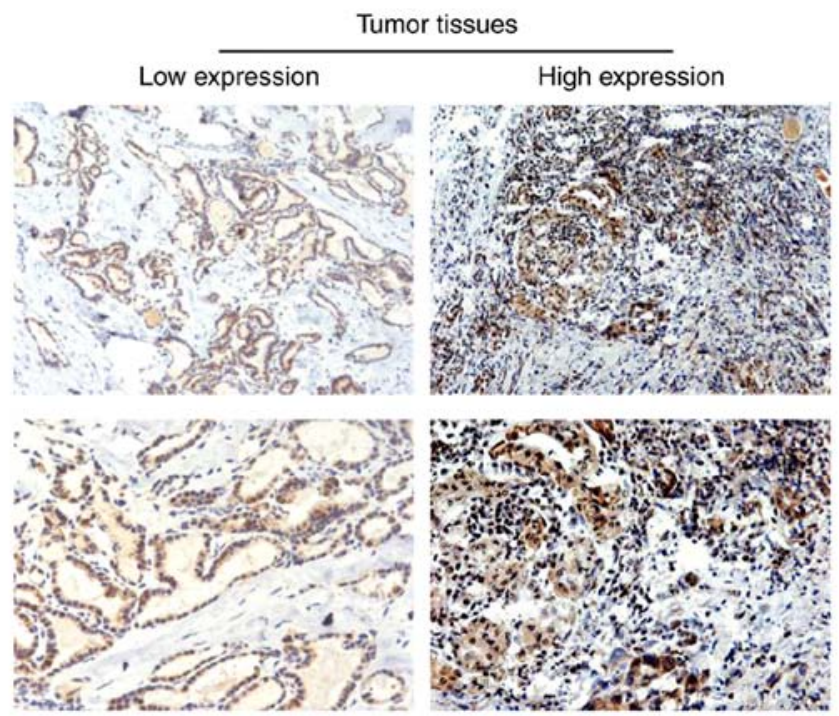

B
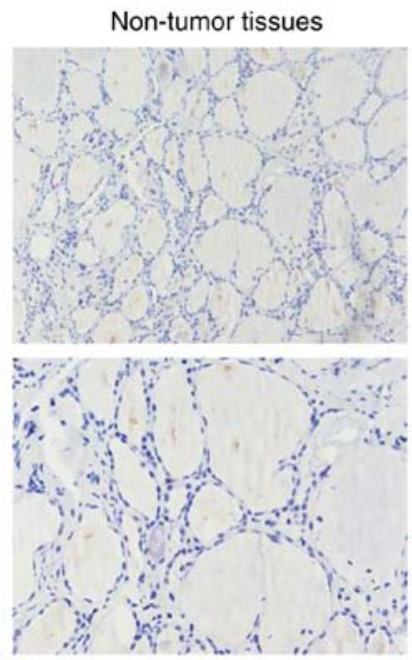

Figure 2. CENPF expression is upregulated in human PTHCA tissues. (A) IHC assays were performed and representative images of CENPF staining in human PTHCA tissues were obtained. (B) IHC assays were conducted and confirmed low CENPF expression in corresponding normal tissues. Magnification for upper images, x100. Magnification for lower images, x200. CENPF, centromere protein F; PTHCA, papillary thyroid cancer; IHC, immunohistochemical.

A

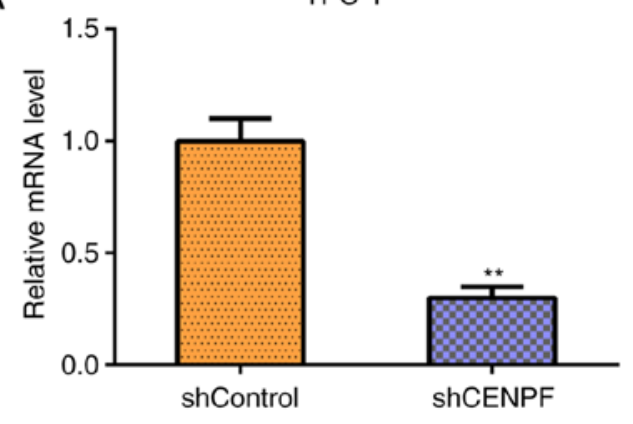

B

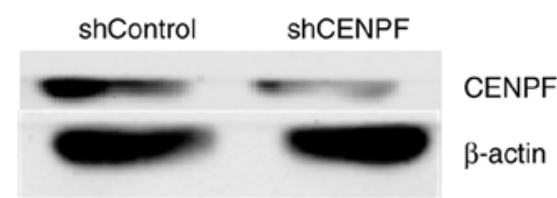

TPC-1

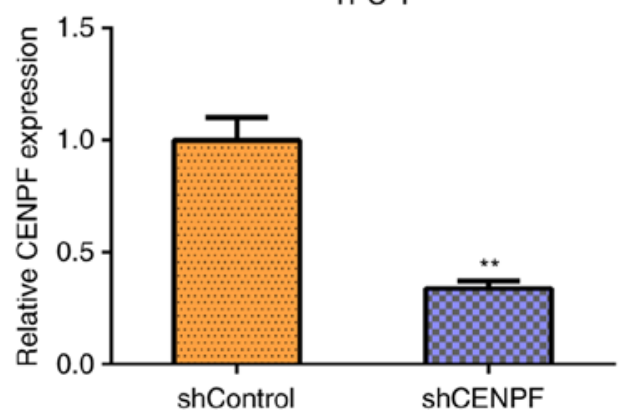

KTC-1

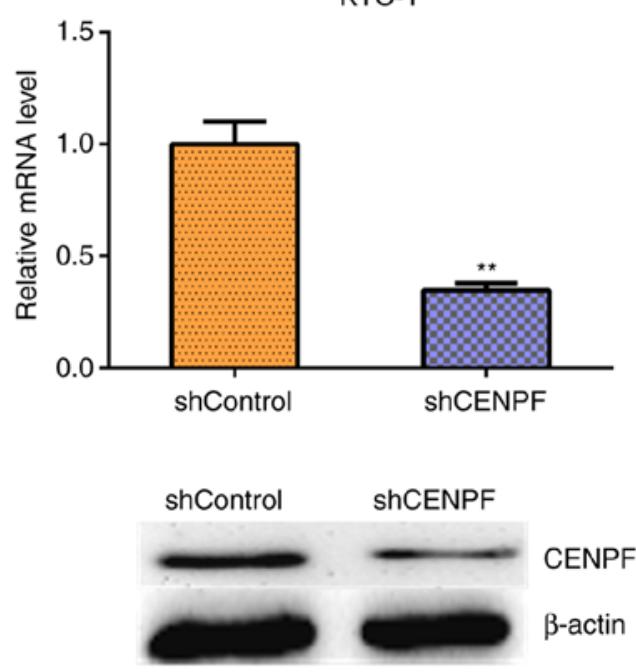

KTC-1

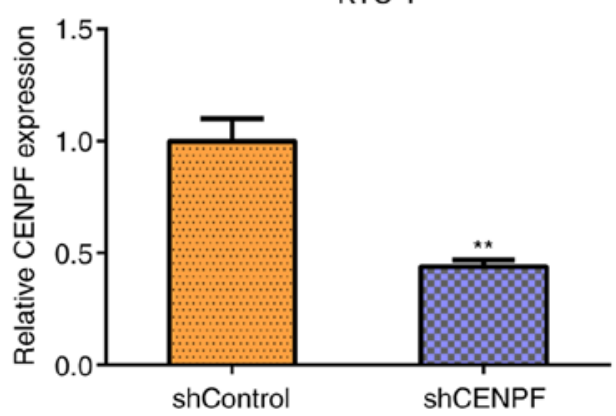

Figure 3. CENPF expression is decreased in TPC-1 and KTC-1 human PTHCA cells as evidenced by shRNA transfection. (A) Quantitative PCR assays revealed a significant decrease in CENPF mRNA levels following transfection with shRNA plasmids into TPC-1 and KTC-1 cells. (B) Western blot analysis demonstrated decreased CENPF expression following transfection of shRNA plasmids into TPC-1 and KTC-1 cells. Data are presented as the mean \pm standard deviation. ${ }^{* *} \mathrm{P}<0.01$ vs. shControl. CENPF, centromere protein F; PTHCA, papillary thyroid cancer; sh, short hairpin.

CENPF promotes the tumor growth of PTHCA cells in vivo. Whether CENPF promoted tumor growth in PTHCA cells was assessed using an animal model to further investigate the effects of CENPF on PTHCA progression. TPC-1 cells 

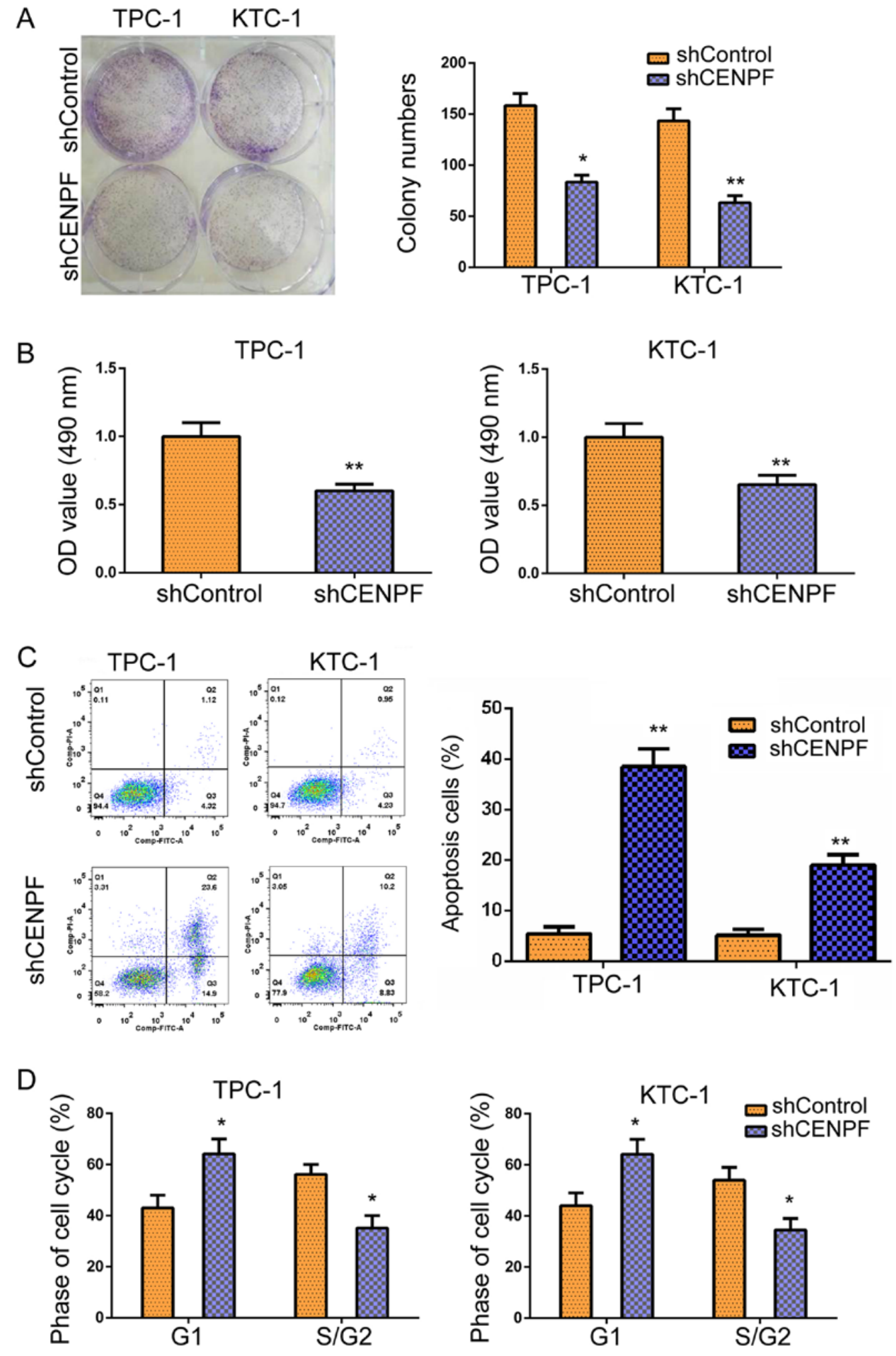

Figure 4. CENPF affects the proliferation, apoptosis and the cell cycles of PTHCA cells in vitro. (A) After TPC-1 and KTC-1 cells were transfected with control or CENPF shRNA plasmids, colony formation assays were performed, and colony numbers were counted. (B) MTT assays demonstrated decreased OD values at $570 \mathrm{~nm}$ following CENPF depletion. (C) FCM assays were performed on TPC-1 and KTC-1 cells and the effects of CENPF on cell apoptosis were detected. (D) FCM assays were performed using TPC-1 and KTC-1 cells following transfection with control or CENPF shRNA plasmids and the effects of CENPF on the cell cycle were detected. Data are presented as the mean \pm standard deviation. ${ }^{*} \mathrm{P}<0.05$ and ${ }^{* *} \mathrm{P}<0.01$ vs. shControl. CENPF, centromere protein F; PTHCA, papillary thyroid cancer; sh, short hairpin; OD, optical density; FCM, flow cytometry.

infected with control or CENPF shRNA lentivirus were subcutaneously injected into nude mice. At 15 days post-injection, tumors began to form, and tumor volumes were measured every 3 days. After 30 days, all tumors were isolated and photographed. Representative images of tumors were presented on the left and tumor growth curves on the right in Fig. 5A. 
A

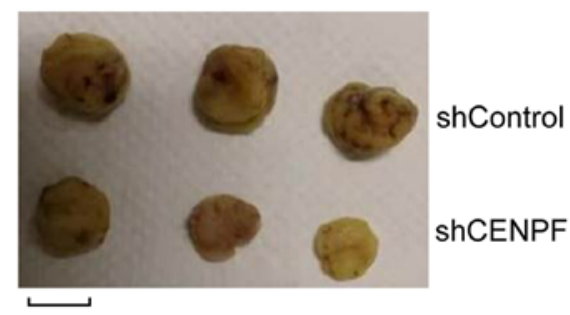

B

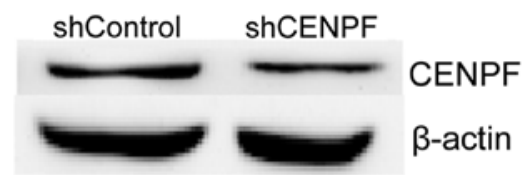

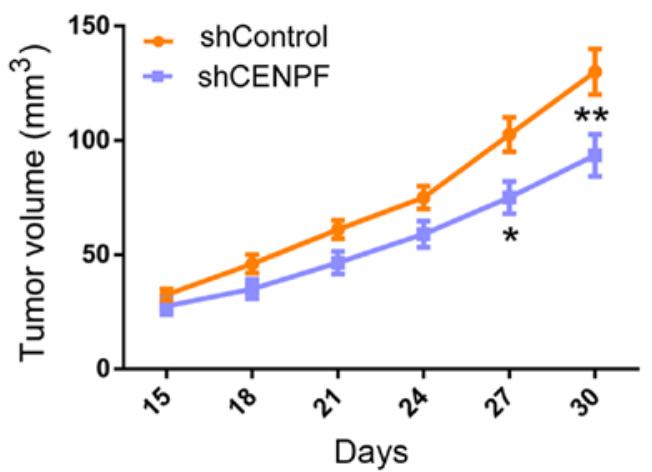

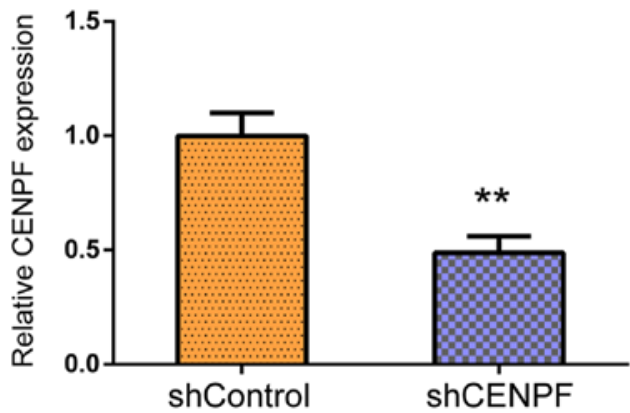

Figure 5. CENPF stimulates tumor growth of PTHCA cells in mice. (A) TPC-1 cells infected with control or CENPF shRNA lentivirus were subcutaneously injected into nude mice. At 15 days post-injection, tumors began to form. Tumor volumes were measured every 3 days. After 30 days, tumors were excised, and the growth curves were analyzed based on the average volume of tumors in the CENPF depletion and control groups. Scale bar, 5 mm. (B) western blotting analysis was performed to analyze the expression levels of CENPF in control or CENPF-depleted tumors. Data are presented as the mean \pm standard deviation. ${ }^{*} \mathrm{P}<0.05$ and ${ }^{* *} \mathrm{P}<0.01$ vs. shControl. CENPF, centromere protein F; PTHCA, papillary thyroid cancer; sh, short hairpin.

Tumor volumes in the CENPF-depleted groups were significantly decreased compared with control groups. Furthermore, western blotting was performed to examine CENPF expression in tumor tissues in control or CENPF-depleted groups. The results demonstrated that CENPF expression levels were decreased in CENPF-depleted tumors (Fig. 5B). These results indicated that CENPF promoted tumor growth of PTHCA cells in vivo.

\section{Discussion}

PTHCA is one of the most common endocrine malignancies and worldwide incidence of PTHCA has markedly increased since 2010 (21). PTHCA is the most common subtype of THCA and accounts for $\sim 85 \%$ of PTHCA cases and usually has a favorable prognosis (21). However, certain patients exhibit high risk for tumor recurrence or death (22). Due to the high metastatic rate of cancer, advances in targeted therapy are urgently required $(23,24)$. Therefore, novel treatments methods, such as targeted therapy, need to be developed to improve the survival rates of PTHCA (25). The results of the current study demonstrated high CENPF expression in human PTHCA tissues compared with corresponding normal tissues. Furthermore, CENP expression was associated with patient prognosis and clinicopathological features. The results indicated the involvement of CENPF in PTHCA progression and suggested that CENPF acted as a promising molecular target for PTHCA treatment.

Colony formation and MTT assays further confirmed that CENPF contributed to the proliferation and cytotoxicity of PTHCA cells in vitro. Furthermore, FCM assays demonstrated that CENPF altered PTHCA cell apoptosis and regulated the cell cycles of PTHCA cells. These results indicated that the depletion of CENPF induced various anti-tumor effects on PTHCA and developing inhibitors targeting CENPF may be an effective therapy to treat this disease. Previous studies have demonstrated that CENPF was abnormally expressed and altered the progression of various types of tumors (13,17,26-29). For instance, CENPF was associated with the prognosis of NSCLC and was associated with tumor bone metastasis of breast cancer (19). Additionally, CENPF was regulated by cycling B1 and contributed to the proliferation and metastasis of gastric cancer (13). Furthermore, lymphoid-specific helicase may also promote the growth and invasion of $\mathrm{HCC}$ by targeting CENPF (13). These previous studies, together with the results of the current study, confirmed the critical role of CENPF in cancer progression. Future studies should be conducted to determine whether CENPF affects the migration and invasion of gastric cancer cells in vitro and tumor metastasis in vivo in mice models.

Through a series of assays, the current study demonstrated that CENPF affected the proliferation and apoptosis of PTHCA cells in vitro. Consistently, in vivo data further confirmed that CENPF affected tumor growth in mice. As the results of the current study confirmed this conclusion, the present study hypothesized that CENPF altered the progression of PTHCA by regulating cell proliferation and apoptosis.

Several previous studies have indicated that CENPF may affect cancer progression through metabolism regulation $(16,18)$. Furthermore, CENPF depletion remodeled prostate cancer cells by altering cellular metabolism (16). Another previous study reported that CENPF modulated 
cancer metabolism by mediating the pyruvate kinase M2 phosphorylation pathway (18). Future studies should clarify whether CENPF promotes PTHCA progression via this signaling pathway.

CENPF is also known as a microtubule binding protein and regulates several microtubule-dependent cellular processes, including mitosis $(9,28)$. A previous study demonstrated that CENPF promoted chromosome segregation and contributed to cell cycle progression (28). Consistent with these previous findings, the current study demonstrated that CENPF depletion induced cell cycle arrest in PTHCA cells; this may be by altering microtubule dynamics and mitosis. The present study also showed that CENPF depletion stimulated PTHCA cell apoptosis. However, whether CENPF exerts its effects through the regulation of microtubules requires further study.

In conclusion, the current study reported enhanced expression of CENPF in human PTHCA tissues. CENPF expression was associated with prognosis and certain clinicopathological features in patients with PTHCA, including T stage and intraglandular dissemination. Further assays confirmed the effect of CENPF on PTHCA cell proliferation and apoptosis in vitro and in vivo tumor growth in mice. Therefore, CENPF may be a promising therapeutic target for PTHCA treatment.

\section{Acknowledgements}

Not applicable.

\section{Funding}

No funding was received.

\section{Availability of data and materials}

The datasets used and/or analyzed during the current study are available from the corresponding author on reasonable request.

\section{Authors' contributions}

YH, SX and YB performed the molecular biology experiments and drafted the manuscript. KC, CD, SL and WZ participated in study design and performed statistical analysis. YB, YH, $\mathrm{SX}, \mathrm{KC}, \mathrm{CD}, \mathrm{SL}$ and $\mathrm{WZ}$ conceived and designed the study and aided in drafting the manuscript. All authors read and approved the final manuscript.

\section{Ethics approval and consent to participate}

All procedures performed in the current study were approved by the Ethics Committee of Binzhou Medical University Hospital, Binzhou, China. Written informed consent was obtained from all patients or their families.

\section{Patient consent for publication}

Not applicable.

\section{Competing interests}

The authors declare that they have no competing interests.

\section{References}

1. Su A, Zhao W, Wu W, Wei T, Ruan M, Li Z and Zhu J: The association of preoperative thyroid-stimulating hormone level and the risk of differentiated thyroid cancer in patients with thyroid nodules: A systematic review and meta-analysis. Am J Surg 220: 634-641, 2020.

2. Shao W, Kuhn C, Mayr D, Ditsch N, Kailuweit M, Wolf V, Harbeck N, Mahner S, Jeschke U, Cavaillès V and Sixou S: Cytoplasmic and nuclear forms of thyroid hormone receptor $\beta 1$ are inversely associated with survival in primary breast cancer. Int J Mol Sci 21: 330, 2020.

3. Qiu K, Xie Q, Jiang S and Lin T: Silencing of DJ-1 reduces proliferation, invasion, and migration of papillary thyroid cancer cells in vitro, probably by increase of PTEN expression. Int J Clin Exp Pathol 12: 2046-2055, 2019.

4. Alghamdi M, Alkhamis WH, Jamjoom D, Al-Nafisah G, Tahir A and Abdouelhoda M: Expanding the phenotype and the genotype of Stromme syndrome: A novel variant of the CENPF gene and literature review. Eur J Med Genet 63: 103844, 2020.

5. Fowler KJ, Saffery R, Irvine DV, Trowell HE and Choo KH: Mouse centromere protein F (Cenpf) gene maps to the distal region of chromosome 1 by interspecific backcross analysis. Cytogenet Cell Genet 82: 180-181, 1998.

6. Ozkinay F, Atik T, Isik E, Gormez Z, Sagiroglu M, Sahin OA, Corduk N and Onay H: A further family of Stromme syndrome carrying CENPF mutation. Am J Med Genet A 173: 1668-1672, 2017.

7. Waters AM, Asfahani R, Carroll P, Bicknell L, Lescai F, Bright A, Chanudet E, Brooks A, Christou-Savina S, Osman G, et al: The kinetochore protein, CENPF, is mutated in human ciliopathy and microcephaly phenotypes. J Med Genet 52: 147-156, 2015.

8. Zhou CJ, Wang XY, Han Z, Wang DH, Ma YZ and Liang CG: Loss of CENPF leads to developmental failure in mouse embryos. Cell Cycle 18: 2784-2799, 2019.

9. Toralova T, Susor A, Nemcova L, Kepkova K and Kanka J: Silencing CENPF in bovine preimplantation embryo induces arrest at 8-cell stage. Reproduction 138: 783-791, 2009.

10. Shi J, Zhang P, Liu L, Min X and Xiao Y: Weighted gene coexpression network analysis identifies a new biomarker of CENPF for prediction disease prognosis and progression in nonmuscle invasive bladder cancer. Mol Genet Genomic Med 7: e982, 2019.

11. Kim HE, Kim DG, Lee KJ, Son JG, Song MY, Park YM, Kim JJ, Cho SW, Chi SG, Cheong HS, et al: Frequent amplification of CENPF, GMNN and CDK13 genes in hepatocellular carcinomas. PLoS One 7: e43223, 2012.

12. Pooley RD, Moynihan KL, Soukoulis V, Reddy S, Francis R, Lo C, Ma LJ and Bader DM: Murine CENPF interacts with syntaxin 4 in the regulation of vesicular transport. J Cell Sci 121: 3413-3421, 2008.

13. Chen EB, Qin X, Peng K, Li Q, Tang C, Wei YC, Yu S, Gan L and Liu TS: HnRNPR-CCNB1/CENPF axis contributes to gastric cancer proliferation and metastasis. Aging (Albany NY) 11: 7473-7491, 2019.

14. Göbel C, Özden C, Schroeder C, Hube-Magg C, Kluth M, Möller-Koop C, Neubauer E, Hinsch A, Jacobsen F, Simon R, et al: Upregulation of centromere protein $\mathrm{F}$ is linked to aggressive prostate cancers. Cancer Manag Res 10: 5491-5504, 2018.

15. Li R, Wang X, Zhao X, Zhang X, Chen H, Ma Y and Liu Y: Centromere protein $\mathrm{F}$ and Forkhead box M1 correlation with prognosis of non-small cell lung cancer. Oncol Lett 19: 1368-1374, 2020

16. Shahid M, Kim M, Lee MY, Yeon A, You S, Kim HL and Kim J: Downregulation of CENPF remodels prostate cancer cells and alters cellular metabolism. Proteomics 19: e1900038, 2019.

17. Aytes A, Mitrofanova A, Lefebvre C, Alvarez MJ, Castillo-Martin M, Zheng T, Eastham JA, Gopalan A, Pienta KJ, Shen MM, et al: Cross-species regulatory network analysis identifies a synergistic interaction between FOXM1 and CENPF that drives prostate cancer malignancy. Cancer Cell 25: 638-651, 2014.

18. Shahid M, Lee MY, Piplani H, Andres AM, Zhou B, Yeon A, Kim M, Kim HL and Kim J: Centromere protein F (CENPF), a microtubule binding protein, modulates cancer metabolism by regulating pyruvate kinase M2 phosphorylation signaling. Cell Cycle 17: 2802-2818, 2018.

19. Sun J, Huang J, Lan J, Zhou K, Gao Y, Song Z, Deng Y, Liu L, Dong Y and Liu X: Overexpression of CENPF correlates with poor prognosis and tumor bone metastasis in breast cancer. Cancer Cell Int 19: 264, 2019. 
20. Livak KJ and Schmittgen TD: Analysis of relative gene expression data using real-time quantitative PCR and the 2(-Delta Delta C(T)) method. Methods 25: 402-408, 2001.

21. Mariniello RM, Orlandella FM, Stefano AE, Iervolino PLC, Smaldone G, Luciano N, Cervone N, Munciguerra F, Esposito S, Mirabelli P and Salvatore G: The TUSC2 tumour suppressor inhibits the malignant phenotype of human thyroid cancer cells via SMAC/DIABLO Protein. Int J Mol Sci 21: 702, 2020.

22. Moreno I, Hirsch D, Duskin-Bitan H, Diker-Cohen T, Shimon I and Robenshtok E: Response to therapy assessment in intermediate-risk thyroid cancer patients-is rhTSH stimulation required? Thyroid 30: 863-870, 2020.

23. Yang Y, Zhao Z, Xie CW and Zhao Y: Dual-targeting liposome modified by glutamic hexapeptide and folic acid for bone metastatic breast cancer. Chem Phys Lipids 228: 104882, 2020.

24. Zhao Z, Zhao Y, Xie CW, Chen CQ, Lin D, Wang S, Lin D, Cui X, Guo Z and Zhou J: Dual-active targeting liposomes drug delivery system for bone metastatic breast cancer: Synthesis and biological evaluation. Chem Phys Lipids 223: 104785, 2019.

25. Qiu ZL, Shen CT, Sun ZK, Song HJ, Zhang GQ and Luo QY: Lung metastases from papillary thyroid cancer with persistently negative thyroglobulin and elevated thyroglobulin Antibody levels during radioactive iodine treatment and follow-up: Long-term outcomes and prognostic indicators. Front Endocrinol (Lausanne) 10: 903, 2019.
26. LinSC,KaoCY,LeeHJ,CreightonCJ,IttmannMM,TsaiSJ,TsaiSYand Tsai MJ: Dysregulation of miRNAs-COUP-TFII-FOXM1-CENPF axis contributes to the metastasis of prostate cancer. Nat Commun 7: $11418,2016$.

27. Lokody I: Signalling: FOXM1 and CENPF: Co-pilots driving prostate cancer. Nat Rev Cancer 14: 450-451, 2014.

28. Mahmoud AD, Ballantyne MD, Miscianinov V, Pinel K, Hung J, Scanlon JP, Iyinikkel J, Kaczynski J, Tavares AS, Bradshaw AC, et al: The human-specific and smooth muscle cell-enriched LncRNA SMILR promotes proliferation by regulating mitotic CENPF mRNA and drives cell-cycle progression which can be targeted to limit vascular remodeling. Circ Res 125: 535-551, 2019.

29. Dai Y, Liu L, Zeng T, Zhu YH, Li J, Chen L, Li Y, Yuan YF, Ma $S$ and Guan XY: Characterization of the oncogenic function of centromere protein $\mathrm{F}$ in hepatocellular carcinoma. Biochem Biophys Res Commun 436: 711-718, 2013.

This work is licensed under a Creative Commons Attribution-NonCommercial-NoDerivatives 4.0 International (CC BY-NC-ND 4.0) License. 